\title{
Dose Characteristics of Radiation-Induced (I-131) Thyroid Cancer
}

\author{
Stojarov $\mathrm{AN}^{1 *}$ and Khrustalev VV ${ }^{2}$ \\ ${ }^{1}$ Department of Radiation Medicine and Ecology, Belarusian State Medical University, Minsk, Belarus \\ ${ }^{2}$ Department of General Chemistry, Belarusian State Medical University, Minsk, Belarus
}

*Corresponding author: Stojarov AN, Department of Radiation Medicine and Ecology, Belarusian State Medical University, Minsk, Belarus

\section{ARTICLE INFO}

Received: 蔧 May 28, 2021

Published: 絊 June 10, 2021

Citation: Stojarov AN, Khrustalev VV. Dose Characteristics of Radiation-Induced (I131) Thyroid Cancer. Biomed J Sci \& Tech Res 36(3)-2021. BJSTR. MS.ID.005848.

Keywords: I-131; Chernobyl Accident; Thyroid Cancer; Absorbed Dose

\section{ABSTRACT}

Analysis of absorbed doses by the Thyroid Gland (TG) of residents of the regions of Belarus affected by the Chernobyl accident showed that the highest doses absorbed by this organ (600-700 mGy) were received by infants and children, who in April 1986 were in the age from 0 to 4 years old. These doses did not differ much between men and women. The curve of absorbed dose values versus age is described well by the exponential dependence. The incidence of Thyroid Cancer (TC) in age groups of both sexes (from 0 to 19 years), depending on the absorbed dose by the TG, was characterized by a linear relationship with a coefficient of determination equal to 0.89 . Persons from the younger age group (0-4 years), who received the highest absorbed doses, had a higher morbidity. Persons from the age group of 15-18 years old at the time of an accident received lower doses by their TG and demonstrated lower incidence of TC. Extrapolation of the direct dependence of the latent period of cancer on the received absorbed dose in those age groups suggests that the shortened latent period of this disease recorded in Belarus may be associated with the formation of very high doses absorbed by TG (more than $1.2 \mathrm{~Gy}$ ) in some children from affected areas.

\section{Introduction}

The first scientifically confirmed stochastic effect after the Chernobyl accident was an increase in the incidence of Thyroid Cancer (TC) among children, first in Belarus, and then in Ukraine and the Russian Federation [1-3]. This phenomenon was completely unpredictable, since the classical ideas about the immediate medical consequences predicted the increase in the number of cases of acute leukemia. However, it should be noted that the nature of the radiation effect on the population can play an important role in the development of the consequences. Thus, during the bombing of Hiroshima and Nagasaki (Japan), external exposure was the dominant effect on the population. While at the early stage of the accident at the Chernobyl nuclear power plant, the first dose-forming factor was associated with the emission of a mixture of iodine radionuclides (I-129, I-131, I-133, I-135 etc.).
One of the ways of the intake of those radionuclides was inhalation. Moreover, as a result of sedimentation from a radioactive cloud, iodine radionuclides contaminated the soil and, due to the functioning of trophic chains, they reached the human body, accumulating in the tropic organ - the Thyroid Gland (TG). In the parenteral route of the intake, whole milk played an important role. In addition to iodine, Cs-137, Cs-134, and Sr-90 radionuclides that contaminated the soil contributed to the formation of both external and internal irradiation. The fact of such a short latent period of TC, that was characteristic to the children, is alarming. The growth of incidence of this disease had begun to be recorded 4-5 years after the accident, i.e. since 1990 [1]. The reasons for this are not clear yet. In this regard, additional studies of this phenomenon are needed for the better understanding of medical consequences of the Chernobyl accident, which is what this work is devoted to. 


\section{Materials and Methods}

As the material for this study we took the data on the incidence of TC from the Belarusian cancer registry for 1986-2016 for rural residents of two districts of the Brest region (Luninets and Stolin districts) affected by the Chernobyl accident [4]. Residents of this area fell under the "iodine blow", i.e. a radioactive cloud passed over the territory of their residence in the night of April 26, 1986. As a result, the territory of the two districts was contaminated by the I-131 in the range from 15 to $100 \mathrm{Ci} / \mathrm{km} 2$. The cohort from the cancer register of two districts included 235 persons, of whom 183 were women and 52 were men. The study included only rural residents of the mentioned areas, who at the time of the accident were in the age between 0 and 19 years old. Since in the previous analysis of TC in residents affected by the Chernobyl accident, which was carried out by UNSCEAR experts in 2016, age groups were identified, in this study we used the same approach and the following age groups were identified in the studied cohort at the time of the accident at the Chernobyl nuclear power plant: 0-4 years, 5-9 years, 10-14 years and 15-18 years [5]. The composition of age groups is presented in Table 1. Doses absorbed by the TG were provided by the head of the laboratory for reconstructing radiation doses of the population of the State Research Center of the Federal Medical Biophysical Center named after I.A. Burnazyan FMBA of Russia Doctor of Technical Sciences, Shinkarev S.M. Doses for children of various ages and adults were calculated using a semiempirical model from the 2004 iteration. Statistical data processing was performed using the Statistic 10.0 (Statsoft Inc., Australia) and SigmaPlot 13 (Systat Software Inc, Germany) application programs. The reliability of the results was assessed using the SigmaPlot program, and the value of $\mathrm{p}<0.05$ considered as the evidence of the existence of significant difference. This study was approved by the Ethics Commission of the Belarusian Association of Physicians (10.12.2020).
Table 1: The age groups of persons in selected districts from the Belarusian Cancer Register.

\begin{tabular}{|c|c|c|c|}
\hline \multirow{2}{*}{ Age group } & \multicolumn{3}{|c|}{ Stolin and Luninets districts } \\
\cline { 2 - 4 } & Both sexes & Female & Male \\
\hline 0-4 years & 44 & 26 & 18 \\
\hline 5-9 years & 23 & 15 & 8 \\
\hline 10-14 years & 23 & 17 & 6 \\
\hline 15-18 years & 19 & 15 & 4 \\
\hline >18 years & 126 & 110 & 16 \\
\hline Total & 235 & 183 & 52 \\
\hline
\end{tabular}

\section{Results}

As indicated above, the intensity of the irradiation plays a role in the manifestation of radiation effects, i.e. the values of the formed doses due to the incorporation of I-131. In this case we are talking about the absorbed doses by the TG of the inhabitants of the abovementioned areas. Figure 1 shows the data on the calculation of absorbed doses by the TG of victims from the entire cohort, as well as men and women. It can be seen that the distribution of absorbed doses by their magnitude differs slightly in men and women, especially in older age groups. Meanwhile, in the age group of 0-4 years, women received slightly higher doses, while in the group of 5-9 years the situation was reversed. However, these differences do not exceed $10 \%$. Considering the values of absorbed doses, it can be noted that in general, especially with regard to older age groups, their values coincide with the data that were previously reported for residents of Belarus [6,7]. Nevertheless, in the age group of 0-4 years, one can see rather high average values of absorbed doses by the TG. Their spread in this group was quite large: from 352 to 1347 mGy. These data are understandable from the point of view that the tissue mass of the TG of infants and young children is much less than that of adults, metabolism is more intense, and milk is the main source of iodine in the body.

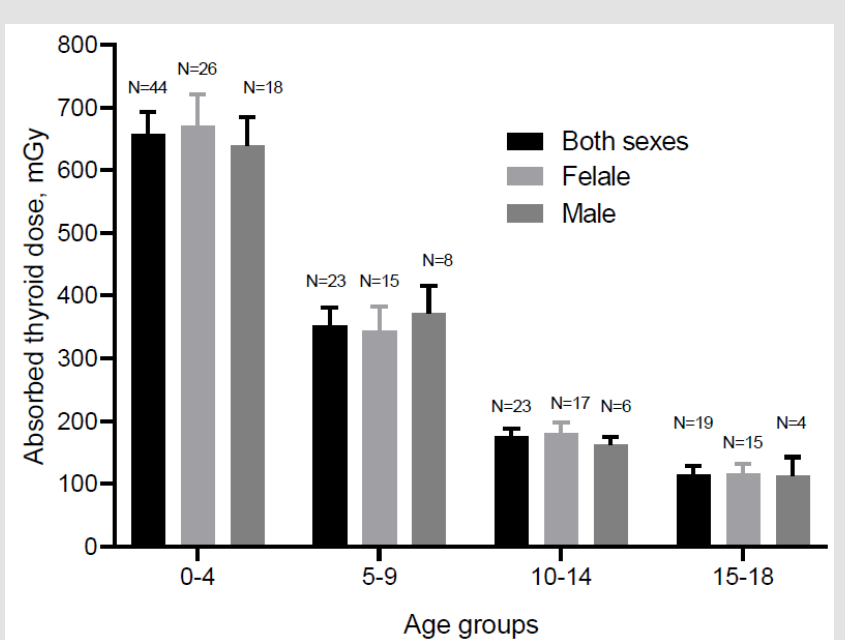

Figure 1: The values of absorbed doses by the TG in the age groups of victims of the Brest region included in the Belarusian cancer registry. 
These results are well illustrated by the curve shown in Figure 2. The dependence of the dose on the age is well described by the exponential curve and for persons of the age from 0 to 10 years it is characterized by a steeper segment. Considering the effect of doses absorbed by TG received during an accident, it is of a great interest to clarify the dose patterns in relation to the age at which TC was diagnosed (Figure 3). It can be seen that in age groups a linear relationship with a coefficient of determination of 0.98 is revealed between those parameters. Moreover, if the victim was exposed to radiation at the age of 0 to 4 years, TC was detected at the age of about 17 years. If the irradiation took place at the age of 15-18 years, then this pathology was recorded much later, at the age of 42 years. Consideration of the relationship between the latent period of the TC and the absorbed dose by this organ in the selected age groups showed that these parameters are related to each other by a rather complex relationship (Figure 4). Persons from the youngest age group (0-4 years) were characterized by high values of the absorbed dose (more than $0.6 \mathrm{~Gy}$ ) and the shortest latency period of 16 years. Persons from older age groups were characterized by lower absorbed thyroid doses and longer latent periods of oncological pathology. So, for example, in the 15-18 year old group, the absorbed doses only slightly exceeded the value of $0.1 \mathrm{~Gy}$, but the TC latency was approaching 25 years.

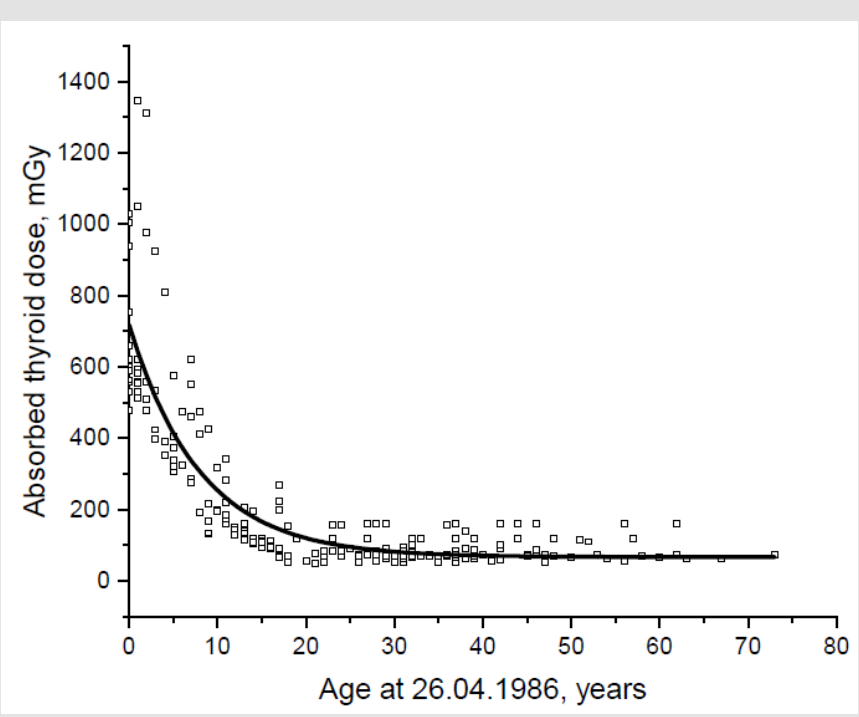

Figure 2: Distribution of absorbed doses by the TG of the affected persons from Stolin and Luninets districts included in the cancer registry.

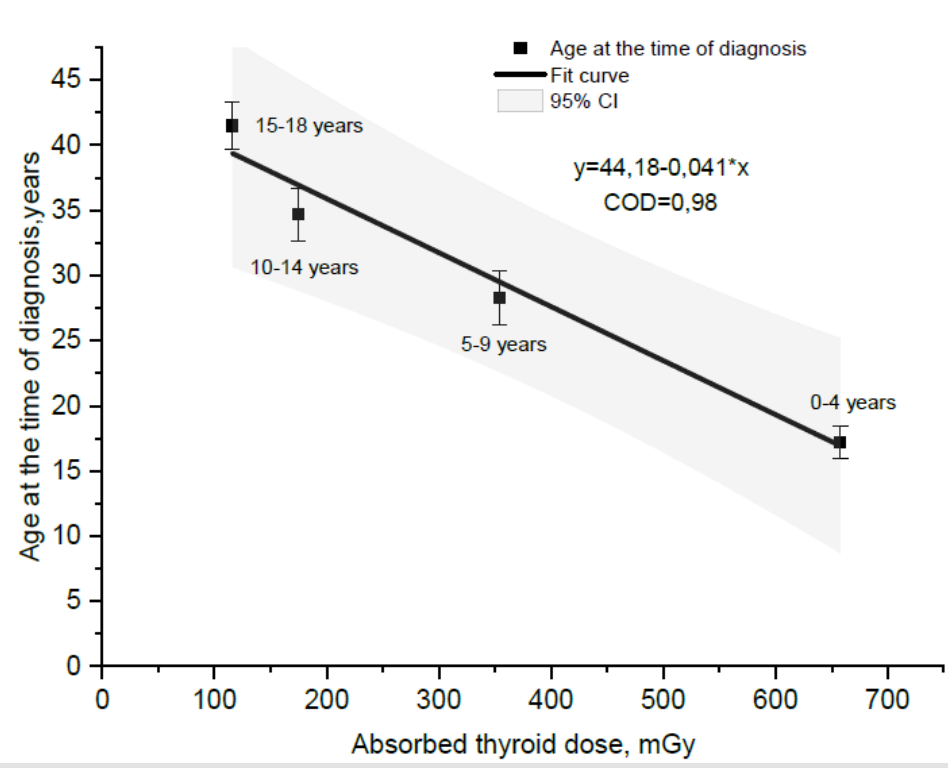

Figure 3: The relationship between the dose received by the TG due to the incorporation of I-131 and the age at which TC was diagnosed in the studied age groups. 


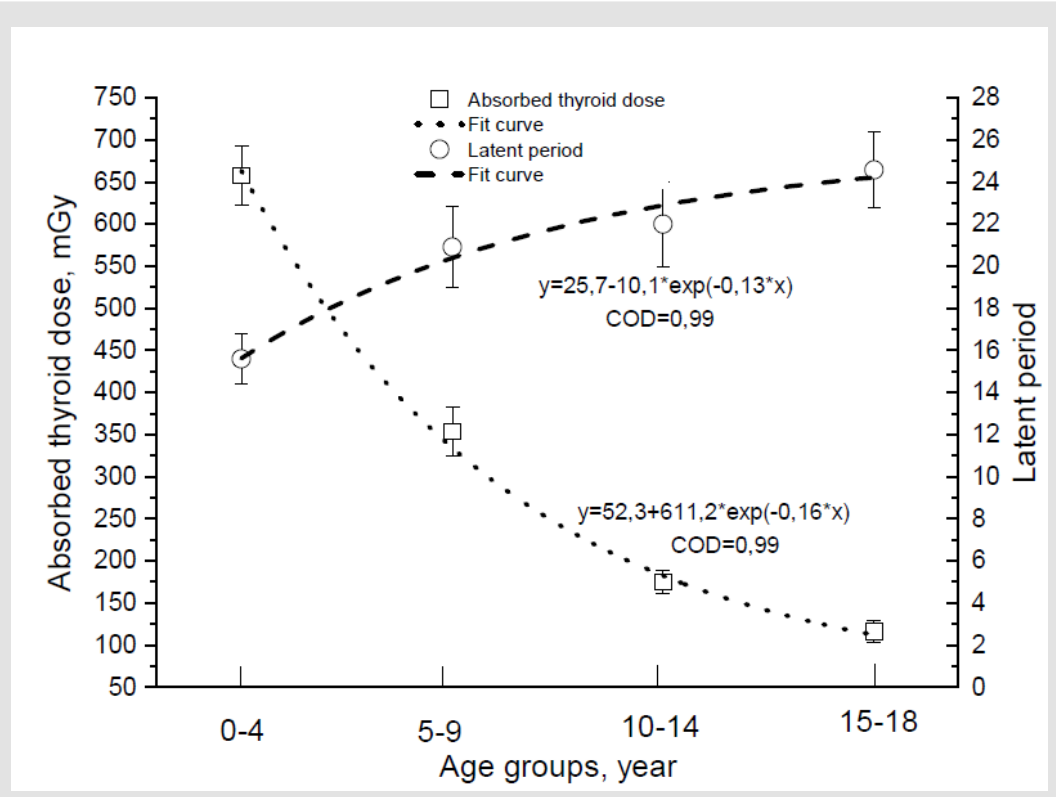

Figure 4: Relationship between the latent period of TC and the absorbed thyroid dose in age groups.

The dependence of morbidity in age groups of both sexes on the absorbed dose by the TG was characterized by a linear relationship with a coefficient of determination of 0.89 (Figure 5). As expected, persons from the younger age group (0-4 years), for whom the highest absorbed thyroid doses were recorded, had a higher morbidity. In persons from the age group of 15-18 years old the doses absorbed by the TG were lower, as well as the incidence of TC. It remains unclear why the fact of the short latency periods in children with TC, which were actually registered in Belarus after the accident, and which were discussed earlier, does not correspond to the data we received. According to the results of the processing of the data from the Belarusian cancer registry, it follows that the latency period, even among representatives of the younger age group, is approaching 16 years (Figure 6). However, if one constructs the dose dependence of the latent periods of TC on a different scale and extrapolates this dependence to the axis of absorbed doses, then observed facts become clear and explainable. According to these data, a short latency period (5-6 years) discovered immediately after the Chernobyl accident in Belarus could be associated with very large values of absorbed doses by the TG. It follows from Figure 6 that this phenomenon could be caused by absorbed doses of more than $1.2 \mathrm{~Gy}$. In addition, initial mutation at the time of exposure can play the role in tumour latency [8].

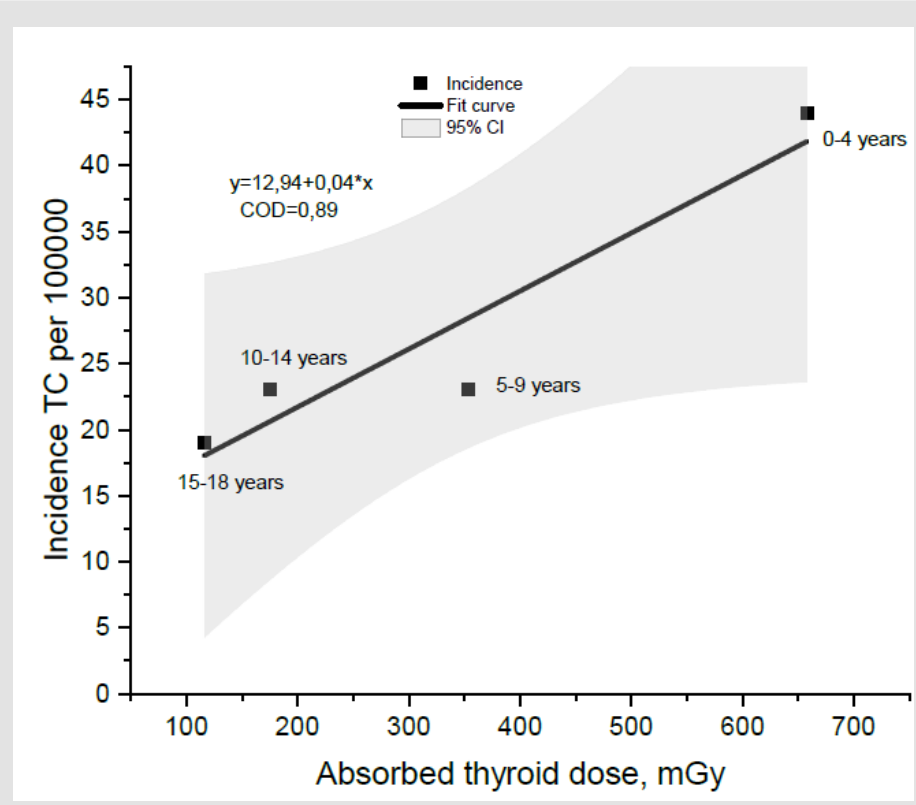

Figure 5: TC incidence in age groups depending on the absorbed thyroid dose. 


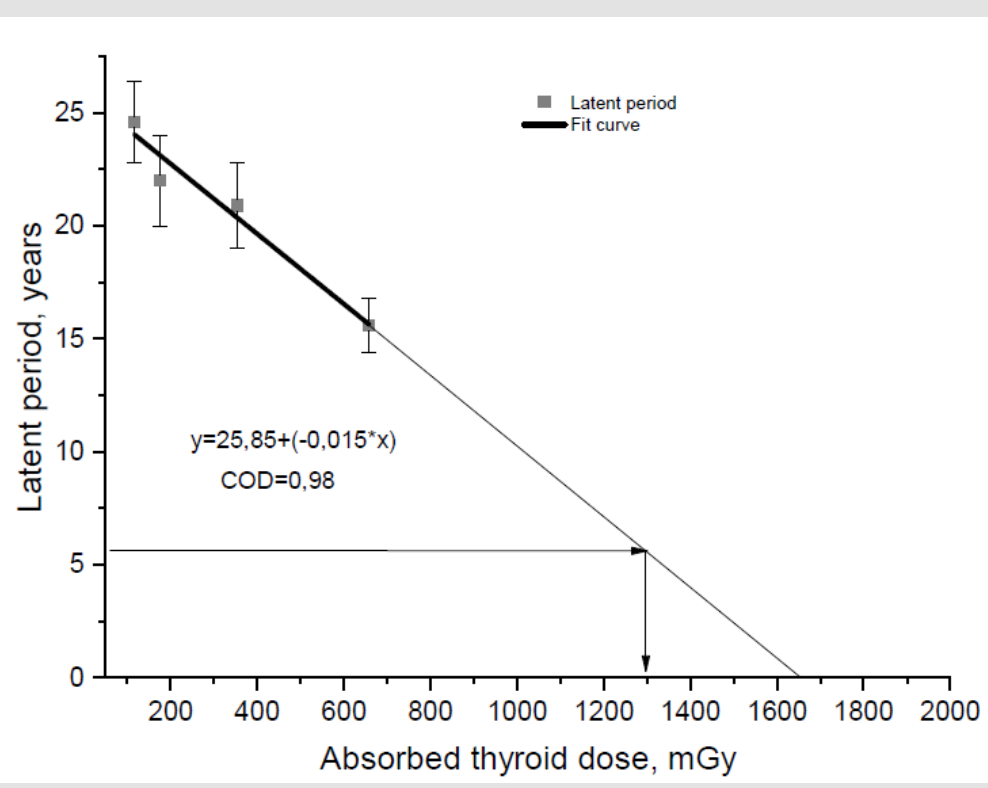

Figure 6: Extrapolation of the data on dose dependence and latent period of TC.

\section{Discussion}

As follows from the presented data, it makes some sense to process the data from the cancer register after the division of all the persons into the age groups. First, the inevitable bias of data is leveled. Secondly, close age implies similar physiological parameters of the organism, in particular, it is very important for processes such as the accumulation of radioactive iodine in the TG, which is accompanied by the formation of absorbed doses, and the value of the absorbed dose in its turn is associated with numerous consequences. Analysis of doses absorbed by the TG due to the incorporation of I-131 shows that the doses for infants and the younger age group were quite high and their formation strongly depends on the age. These results are quite expectable. According to the Belarusian Cancer Registry, in two affected districts of the Brest region, TC cases were characterized by a rather long latency period (at least, 16 years). This was the case for the persons from the youngest age group. The actual data obtained during the medical examination in Belarus immediately after the Chernobyl accident indicate a shorter latency period of 5-6 years. This discrepancy can be explained if we consider the fact that very large doses (more than $1200 \mathrm{mGy}$ ) in individuals of the younger age group can cause a shortening of the latency period. Moreover, even in the sample taken by us from the Belarusian cancer register, as mentioned above, the spread of absorbed doses was very large and included values exceeding 1.0 Gy. It should be considered that in the Gomel region of Belarus, which directly borders with the Chernobyl district of Ukraine (the one with the nuclear power plant), the impact of radiation to the population was expressed to a much greater extent. There, the proportion of high absorbed doses by the
TG should be higher. Hence, it is not surprising that exactly in this region the growth of $\mathrm{TC}$ in children was initially recorded during a short period of time after the Chernobyl accident [1].

\section{Conclusion}

Radiation exposure of the population due to the incorporation of radioactive iodine (I-131) as a result of the accident at the nuclear power plant in the affected areas of the Brest region led to the formation of significant absorbed doses by the TG, especially in infants and children of the younger age group. This was the reason for the subsequent development of TC. An increased frequency of this pathology has been registered in Belarus since 1990. At the same time, the analysis of the data of the Belarusian cancer registry made it possible to discover a longer latency period for the onset of this pathology for children who were from 0 to 4 years old at the time of the accident. This discrepancy can be explained by the formation of very high absorbed doses exceeding the value of 1.2 Gy, at least in some cases, which could be the reason for the early onset of TC.

\section{Acknowledgement}

The authors express their gratitude to the head of the laboratory for reconstructing radiation doses of the population of the State Research Center of the Federal Medical Biophysical Center named after V.I. A.I. Burnazyan FMBA of Russian Federation, Doctor of Technical Sciences, Shinkarev S.M. for data on the calculation of absorbed thyroid doses.

\section{References}

1. Kazakov V, Demidchik E , Astakhova L (1992) Thyroid cancer after Chernobyl. Nature 359(6390): 21. 
2. Ron E (2007) Thyroid Cancer Incidence Among People Living in Areas Contaminated by Radiation From the Chernobyl Accident. Health Phys 93(5): 502-511.

3. (2008) UNSCEAR 2008 Report: "Sources and effects of ionizing radiation". Annex D 1: 219.

4. Stojarov AN, Okeanov AE, Khrustalev VV, Yaumenenka AA (2021) Thyroid cancer in person as a result of the Chernobyl accident. Instant Journal of Hematology and Oncology 2(1): 11.

5. (2018) UNSCEAR (United Nations Scientific Committee on the Effects of Atomic Radiation). New York Evaluation of data on thyroid cancer in regions affected by the Chernobyl accident: A white paper to guide the Scientific Committee's future programme of work, p. 20.

\section{ISSN: 2574-1241}

DOI: 10.26717/BJSTR.2021.36.005849

Stojarov AN. Biomed J Sci \& Tech Res

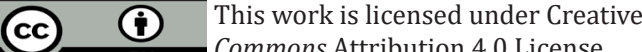

Submission Link: https://biomedres.us/submit-manuscript.php
6. Gavrilin Y, Khrouch V, Shinkarev S (2004) Individual thyroid dose estimation for a casecontrol study of Chernobyl-related thyroid cancer among children of Belarus-part I: 131I, short-lived radioiodines (132I, 133I, 135I), and short-lived radiotelluriums (131MTe and 132Te). Health Phys 86(6): 565-585.

7. Drozdovitch V, Minenko V, Kukhta T (2020) Thyroid Dose Estimates for a Cohort of Belarusian Persons Exposed in Utero and During Early Life to Chernobyl Fallout. Health Phys 118(2): 170-184.

8. Williams ED, Abrosimov A, Bogdanova T (2004) Thyroid carcinoma after Chernobyl latent period, morphology and aggressiveness. British Journal of Cancer 90(11): 2219-2224.

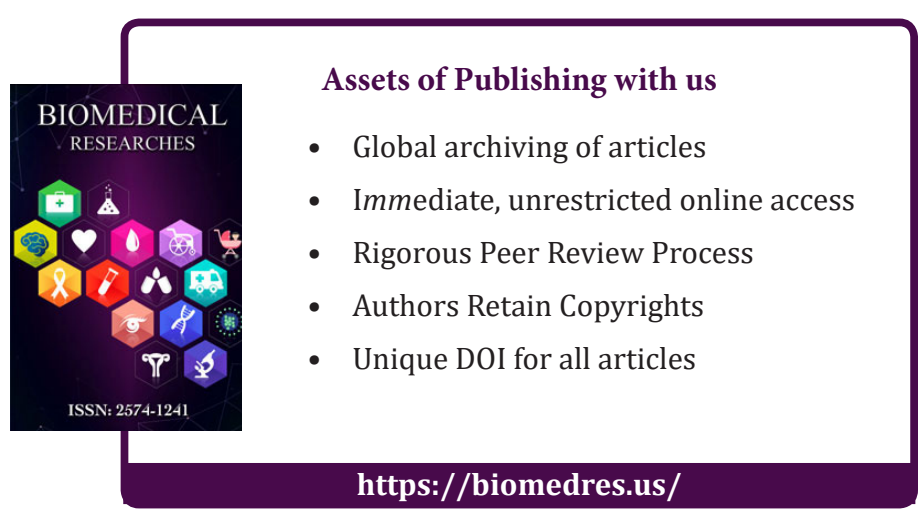

